\title{
E-optimal Design in Irregular BIBD Settings
}

\author{
J. P. Morgan \\ Department of Statistics \\ Brian Reck \\ Virginia Tech \\ Department of Human Genetics \\ University of Pittsburgh \\ Blacksburg, Virginia 24061-0439, USA Pittsburgh, Pennsylvania 15261 USA
}

$14^{\text {th }}$ August 2005

\begin{abstract}
When the necessary conditions for a BIBD are satisfied, but no BIBD exists, there is no simple answer for the optimal design problem. This paper identifies the E-optimal information matrices for any such irregular BIBD setting when the number of treatments is no larger than 100. A- and D-optimal designs are typically not E-optimal. An E-optimal design for 15 treatments in 21 blocks of size 5 is found.
\end{abstract}

Keywords: Balanced incomplete block design; Discrepancy matrix; Design optimality

\section{INTRODUCTION}

A block design setting is an integer triple $(v, b, k)$ specifying the numbers of treatments, blocks, and experimental units per block, respectively, available for an experiment. If these three integers satisfy (i) $v \mid b k$ and $(i i) v(v-1) \mid b k(k-1)$, then $(v, b, k)$ is called a BIBD setting. A balanced incomplete block design (shortly, BIBD) is an assignment of the $v$ treatments to the $b k$ experimental units so that no treatment appears more than once in any block (binarity), each treatment appears on

exactly $r=\frac{b k}{v}$ units (equireplication), and each pair of treatments occurs in $\lambda=\frac{b k(k-1)}{v(v-1)}$ blocks (equiconcurrence).

That $(v, b, k)$ is a BIBD setting is obviously a necessary condition for existence of a BIBD. If $(v, b, k)$ is a BIBD setting but no BIBD exists, then it is called an irregular BIBD setting.

Let $D(v, b, k)$ be the class of all connected designs for the setting $(v, b, k)$, that is, the class of 
designs for which all treatment contrasts are estimable. For any given setting, the statistician's problem is to determine the best member of $D$ according to the relevant criterion or criteria. Criteria are usually formulated as functions of the information matrix $C_{d}$ for $\operatorname{design} d$ :

$$
C_{d}=D\left(r_{d i}\right)-\frac{1}{k} N_{d} N_{d}^{\prime}
$$

where $D\left(r_{d i}\right)$ is the diagonal matrix of replications numbers $r_{d i}, i=1, \ldots, v$ for design $d$, and $N_{d}$ is the $v \times b$ treatment/block incidence matrix with elements $\left(N_{d}\right)_{i j}=n_{d i j}=$ the number of plots assigned treatment $i$ in block $j$ by design $d$. $C_{d}$ is symmetric and nonnegative definite, with rank $v-1$ for $d \in D$. Thus it has eigenvalues

$$
0=z_{d 0}<z_{d 1} \leq \ldots \leq z_{d, v-1}
$$

Among the popular criteria are $A_{d}=\sum_{i=1}^{v-1} z_{d i}^{-1}, D_{d}=-\sum_{i=1}^{v-1} \log \left(z_{d i}\right)$, and $E_{d}=1 / z_{d 1}$. An optimal design with respect to a criterion minimizes that criterion, so that (for instance) an Aoptimal design $d^{*}$ achieves $A_{d^{*}}=\min _{d \in D} A_{d}$. BIBDs, when they exist, are known to enjoy A, D, E, and many other optimalities; see Kiefer (1975).

Denote the binary subclass of $D$ by $M(v, b, k)$ and the subclass of $M$ containing only equireplicate designs by $M_{0}(v, b, k)$. A common strategy in optimal design theory is to use bounds to rule out both nonbinary designs and nonequireplicate designs, then develop techniques to determine the best design in $M_{0}$. Let $I$ denote the identity matrix and $J$ the all-ones matrix. For any $d \in M_{0}$ in an irregular BIBD setting, write $\Delta_{d}=N_{d} N_{d}^{\prime}-r I-\lambda(J-I)$ so that

$$
\begin{aligned}
C_{d} & =r I-\frac{r}{k} I-\frac{\lambda}{k}(J-I)-\frac{1}{k} \Delta_{d} \\
& =\frac{\lambda v}{k}\left(I-\frac{1}{v} J\right)-\frac{1}{k} \Delta_{d}
\end{aligned}
$$

$\Delta_{d}$ is called the discrepancy matrix for design $d$; its diagonal entries are 0 and off-diagonal are $\delta_{d i i^{\prime}}=\sum_{j=1}^{b} n_{d i j} n_{d i^{\prime} j}-\lambda=\lambda_{d i i^{\prime}}-\lambda$, called the pairwise discrepancies for design $d$.

The all-ones vector is the eigenvector of $C_{d}$ corresponding to $z_{d 0}=0$, and likewise is an eigenvector of $\Delta_{d}$ with eigenvalue $x_{d 0}=0$. Consequently any set of eigenvectors for $z_{d 1}, \ldots, z_{d, v-1}$ are each orthogonal to the all-ones vector, are thereby also eigenvectors of $I-\frac{1}{v} J$, and so too of $\Delta_{d}$. Setting $x_{d 0}$ aside, let $x_{d 1} \geq x_{d 2} \geq \ldots \geq x_{d, v-1}$ be the remaining $v-1$ eigenvalues of $\Delta_{d}$ (which may include one or more additional zeros and in any case sum to zero). Then

$$
x_{d i}=\left(\lambda v-z_{d i}\right) / k
$$


$i=1, \ldots, v-1$. For any eigenvalue-based criterion, optimality over $M_{0}$ may be studied through the discrepancy matrices $\Delta_{d}$ rather than the information matrices $C_{d}$.

Morgan and Srivastav (2000) determined a collection of 11 discrepancy matrices with this property: should a design exist with corresponding discrepancy matrix in their list, then any A-optimal and any D-optimal design must have discrepancy matrix in their list. Defining the discrepancy $\delta_{d}$ of design $d$ as the absolute value of one-half the sum of the negative entries in $\Delta_{d}$, these 11 matrices are exactly those with $\delta_{d} \leq 4$. Reck and Morgan (2005) extended Morgan and Srivastav's (2000) result to include all 51 discrepancy matrices with $\delta_{d} \leq 5$. Determining an optimal design depends on solving the existence/nonexistence problems for designs corresponding to at least some of these discrepancy matrices. Reck and Morgan (2005) find an A- and D-optimal design in $D(15,21,5)$ with discrepancy 4 by conducting a constructive search that also rules out existence of all designs with $\delta_{d} \leq 3$ in this setting.

It should be noted here that any permutation applied simultaneously to rows and columns of a discrepancy matrix produces an equivalent discrepancy matrix with the same eigenvalues (this is just a relabelling of treatments). Thus all nonzero rows/columns may be brought to the upper left $s \times s$ submatrix of any $\Delta_{d}$ for some $s$ (depending on $d$ ), and only that $s \times s$ submatrix need be examined, as the eigenvalues of $\Delta_{d}$ are those of the submatrix in addition to $v-s$ zeros. These submatrices are themselves discrepancy matrices for designs with $s$ treatments. More generally they are discrepancy matrices for designs with any $v \geq s$, it being understood that this means they are embedded in a $v \times v$ matrix with all other elements zero. The discrepancy matrices in the lists of the two papers cited above are in fact submatrices of various orders as just described.

Not yet addressed in the literature is the question of E-optimality in irregular BIBD settings. Many well-known A-optimal block designs in settings where equireplication is possible $(v \mid b k)$ are also E-optimal; this includes the BIBDs and many group divisible designs. For irregular BIBD settings, this raises the question of whether or not the E-best discrepancy matrices are among those in the lists of Morgan and Srivastav (2000) or Reck and Morgan (2005). The surprising answer, as shown in section 3 , is no. The optimality tools needed to build this result are developed in section 2. With the E-best discrepancy matrices in hand, an E-optimal design for $D(15,21,5)$ is found in section 4 . Summary remarks are in section 5 . 


\section{E-ORDERING OF DISCREPANCY MATRICES}

An E-optimal design $d^{*}$ satisfies $E_{d^{*}}=\min _{d \in D} \frac{1}{z_{d 1}}$. By virtue of (1), if $d^{*}$ can be shown to lie in $M_{0}$, then

$$
E_{d^{*}}=\min _{d \in M_{0}} \frac{1}{\lambda v-k x_{d 1}} .
$$

In any case, $d^{*}$ is E-optimal over $M_{0}$ if and only if

$$
x_{d^{*} 1}=\min _{d \in M_{0}} x_{d 1}
$$

That is, $d^{*}$ minimizes the maximum eigenvalue of $\Delta_{d}$.

The plan here is to determine the E-best discrepancy matrices, that is, those discrepancy matrices satisfying (3). These lead to E-best designs only if designs in $D$ that are not in $M_{0}$ can be ruled out as E-competitors. This latter task will be disposed of first.

Lemma 1 Let $\bar{d} \in M_{0}$ for the irregular BIBD setting $(v, b, k)$ have discrepancy matrix $\Delta_{\bar{d}}$ with maximum eigenvalue $x_{\bar{d} 1}$. If $x_{\bar{d} 1}<2$ then any E-optimal design must be in $M(v, b, k)$ (that is, must be binary).

Proof Let $d$ be a nonbinary design in $D(v, b, k)$ with E-value $E_{d}$. From the proof of proposition 3.1 of Jacroux (1980b),

$$
E_{d} \geq \frac{k(v-1)}{[r(k-1)-2] v} \geq \frac{k}{\lambda v-2} .
$$

From equation (2), the E-value of $\bar{d}$ is

$$
E_{\bar{d}}=\frac{k}{\lambda v-x_{\bar{d} 1}}
$$

and the result follows.

Lemma 2 Let $\bar{d} \in M_{0}$ for the irregular BIBD setting $(v, b, k)$ have discrepancy matrix $\Delta_{\bar{d}}$ with maximum eigenvalue $x_{\bar{d} 1}$. If $x_{\bar{d} 1}<(k-1)$ then any E-optimal design must be equally replicated.

Proof Let $d$ be any unequally replicated design, and let $\rho_{d}$ be the largest replication shortfall for $d, \rho_{d}=\max _{i}\left(r-r_{d i}\right)$. If $E_{d}$ is the E-value of $d$ then, by Theorem 3.1 of Jacroux (1980a),

$$
E_{d} \geq \frac{(v-1) k}{\left(r-\rho_{d}\right)(k-1) v}=\frac{r k}{\lambda v\left(r-\rho_{d}\right)}
$$


Design $d$ is ruled out if

$$
E_{\bar{d}}=\frac{k}{\lambda v-x_{\bar{d} 1}} \leq \frac{r k}{\lambda v\left(r-\rho_{d}\right)}
$$

or equivalently if

$$
x_{\bar{d} 1}<\frac{\lambda v \rho_{d}}{r}=\left(k-1+\frac{\lambda}{k}\right) \rho_{d}
$$

for which $x_{\bar{d} 1}<(k-1)$ is clearly sufficient.

COROLlary 3 For the irregular BIBD setting $(v, b, k)$, if there exists $\bar{d} \in M_{0}$ having discrepancy matrix among the list of 51 matrices given by Reck and Morgan (2005), then the E-best design in $D(v, b, k)$ must be equireplicate. If there exists $\bar{d} \in M_{0}$ having discrepancy matrix with $x_{\bar{d} 1}<2$, any E-optimal design must be binary as well as equireplicate, that is, must lie in $M_{0}(v, b, k)$.

Proof Since nonexistence of a BIBD implies $k \geq 5$ (see Nandi, 1945, and Hanani, 1961) the first part of the corollary is a simple manner of checking that $x_{\bar{d} 1}<4$ for all 51 of the relevant discrepancy matrices. The corresponding list of $x_{\bar{d} 1}$-values is given in Appendix $\mathrm{A}$, and the largest value is 3.44949 for D51. The second part of the corollary is now immediate from the two preceding lemmas.

In the Reck and Morgan (2005) listing of discrepancy matrices, there are four for which the largest eigenvalue is less than 2, these being (in their labelling) matrices D2, D13, D23, and D5 with respective values 1.73205, 1.87939, 1.902112, 1.93543 (see Appendix A). It is immediately obvious, and contrary to both the A and D behavior, that the E-ordering of discrepancy matrices does not respect the $\delta_{d}$-ordering: the discrepancy values $\delta_{d}$ for these four matrices are respectively $3,5,5,4$. There are another seven matrices (D1, D4, D6, D7, D14, D15, D24) with largest eigenvalues of 2, among which is the sole minimum discrepancy matrix with $\delta_{d}=2$. If 2 is the smallest achievable value of $x_{d 1}$ over $M_{0}$, then any corresponding design is E-optimal, though lemma 1 leaves open the possibility that E-equal competitors lie outside $M_{0}$.

That the E-ordering of discrepancy matrices need not respect the $\delta_{d}$-ordering is further evidenced by the following fact: if $\Delta_{d}$ is a discrepancy matrix with discrepancy value $\delta_{d}$ and maximum eigenvalue $x_{d 1}$, then $I_{n} \otimes \Delta_{d}$ is a discrepancy matrix with discrepancy value $n \delta_{d}$ but still having maximum eigenvalue $x_{d 1}$. Taking $n=\operatorname{int}\left(\frac{v}{6}\right)$, there is a discrepancy matrix of order no more than $v$ with discrepancy $3 n$ that is as good as or better than every discrepancy matrix with positive 
discrepancy less than 6. Even if a design with discrepancy matrix $D 2$ does not exist, as is the case in $(15,21,5)$, there may well exist a design with discrepancy matrix $I_{n} \otimes D 2$. In light of this observation and the results above, the E-question in irregular BIBD settings may be posed thusly: does there exist a discrepancy matrix with maximal eigenvalue less than that of D2 (1.73205)?

This problem will be attacked with a constructive approach in section 3, building up submatrices of discrepancy matrices one row and column at a time until either an E-superior discrepancy matrix is obtained or the current submatrix is eliminated from contention. As each new row/column is added to a submatrix, it is judged for feasibility using the next result.

Lemma 4 Let $\Delta_{d}$ with maximal eigenvalue $x_{d 1}$ be the discrepancy matrix for a design $d \in M_{0}(v, b, k)$, and define $\Delta_{d 11}$ to be the $m \times m, m \leq v$, leading diagonal submatrix of $\Delta_{d}$. Then for any normalized $w_{m \times 1}$,

$$
x_{d 1} \geq\left[1-\frac{\left(\sum w_{i}\right)^{2}}{v}\right]^{-1} w^{T} \Delta_{d 11} w .
$$

Proof Since $\Delta_{d}$ has row and column sums of zero,

$$
x_{d 1}=\max _{\substack{x^{T} x=1 \\ x^{T} 1=0}} x^{T} \Delta_{d} x .
$$

Partition $\Delta_{d}$ as

$$
\Delta_{d}=\left(\begin{array}{cc}
\Delta_{d 11} & \Delta_{d 12} \\
\Delta_{d 21} & \Delta_{d 22}
\end{array}\right)
$$

and consider the vector $y^{T}=\left(w^{T}, 0^{T}\right), w^{T} w=1$, so that $y^{T} \Delta_{d} y=w^{T} \Delta_{d 11} w$. Then, provided $w^{T} 1=0$,

$$
x_{d 1} \geq w^{T} \Delta_{d 11} w .
$$

If $w^{T} 1 \neq 0$, consider $y^{*}=\left(I-\frac{1}{v} J\right) y=y-\frac{1}{v} \sum y_{i} 1=y-\frac{1}{v} \sum w_{i} 1$. Then $y^{* T} 1=0$ and

$$
\begin{aligned}
y^{* T} y^{*} & =y^{T} y-\frac{2}{v}\left(\sum w_{i}\right) y^{T} 1+\frac{1}{v^{2}}\left(\sum w_{i}\right)^{2} 1^{T} 1 \\
& =1-\frac{\left(\sum w_{i}\right)^{2}}{v} \\
& =q \text { (say). }
\end{aligned}
$$

Thus

$$
x_{d 1} \geq \frac{1}{q} y^{* T} \Delta_{d} y^{*}=\frac{1}{q}\left(y-\frac{1}{v} \sum w_{i} 1\right)^{T} \Delta_{d}\left(y-\frac{1}{v} \sum w_{i} 1\right)
$$




$$
\begin{aligned}
& =\frac{1}{q} y^{T} \Delta_{d} y \quad\left(\text { since } 1^{T} \Delta_{d}=0\right) \\
& =\left[1-\frac{\left(\sum w_{i}\right)^{2}}{v}\right]^{-1} w^{T} \Delta_{d 11} w .
\end{aligned}
$$

Building up discrepancy matrices $\Delta_{d}=\left(\delta_{d i i^{\prime}}\right)$ also requires knowing a set of admissible values for the pairwise discrepancies $\delta_{d i i^{\prime}}$. That is the purpose of the next result.

Lemma 5 Let $d \in M_{0}$ for the irregular BIBD setting $(v, b, k)$ have discrepancy matrix $\Delta_{d}$ with maximum eigenvalue $x_{d 1}$. Then

$$
\min _{i \neq i^{\prime}} \delta_{d i i^{\prime}} \geq-x_{d 1}
$$

and

$$
\max _{i \neq i^{\prime}} \delta_{d i i^{\prime}} \leq \frac{v-2}{v} x_{d 1}
$$

Proof By Proposition 3.2 of Jacroux (1980b), $E_{d}$ for any $d$ in $M_{0}$ satisfies, for all $\lambda_{d i i^{\prime}}\left(i \neq i^{\prime}\right)$

$$
E_{d} \geq \frac{k}{r(k-1)+\lambda_{d i i^{\prime}}}
$$

and

$$
E_{d} \geq \frac{(v-2) k}{\left[r(k-1)-\lambda_{d i i^{\prime}}\right] v} .
$$

Since $M_{0}$ is a BIBD setting, $r(k-1)=\lambda(v-1)$. Using the relationships $\lambda_{d i i^{\prime}}=\lambda+\delta_{d i i^{\prime}}$ and $E_{d}=\frac{1}{\lambda v-k x_{d 1}}$, inequality (6) may be rewritten as $\delta_{d i i^{\prime}} \geq-x_{d 1}$ for all $i \neq i^{\prime}$, and, similarly, inequality (7) becomes $\delta_{d i i^{\prime}} \leq \frac{v-2}{v} x_{d 1}$ for all $i \neq i^{\prime}$, establishing (4) and (5).

COROLlary 6 If design $d$ with discrepancy matrix $\Delta_{d}$ is E-better than design $\bar{d}$, then

$$
\min _{i \neq i^{\prime}} \delta_{d i i^{\prime}}>-x_{\bar{d} 1}
$$

and

$$
\max _{i \neq i^{\prime}} \delta_{d i i^{\prime}}<\frac{v-2}{v} x_{\bar{d} 1}
$$

In corollary 6 let $\bar{d}$ be a design having discrepancy matrix $I_{n} \otimes D 2$ for some $n \geq 1$, so that $x_{\bar{d} 1}=1.73205$. Then equaling (relax the inequalities to not be strict) or bettering $\bar{d}$ can only 
be achieved by a design $d$ satisfying $-1 \leq \delta_{d i i^{\prime}} \leq 1$ for all $i \neq i^{\prime}$. Indeed the same statement is true relative to any of the eleven matrices in the Reck and Morgan (2005) listing having maximal eigenvalue no more than 2 .

Before beginning the construction of discrepancy matrices, one last ordering result is needed.

Lemma 7 If $\Delta_{d}$ can be partitioned

$$
\Delta_{d}=\left(\begin{array}{cc}
\Delta_{d 11} & \Delta_{d 12} \\
\Delta_{d 21} & \Delta_{d 22}
\end{array}\right)
$$

so that $\Delta_{d 11}$ is a discrepancy matrix, then $\Delta_{d}$ cannot be E-better than $\Delta_{d 11}$.

PROOF Theorem C.1 on page 225 of Marshall and Olkin (1979) says that $\Delta_{d}$ majorizes $\left(\begin{array}{cc}\Delta_{d 11} & 0 \\ 0 & \Delta_{d 22}\end{array}\right)$, which in turn majorizes $\left(\begin{array}{cc}\Delta_{d 11} & 0 \\ 0 & 0\end{array}\right)$, from which the E-ordering is immediate.

\section{Construction of E-Best discrepancy matrices}

The goal here is to find any and all discrepancy matrices that are at least as good, in the E sense, as $I_{n} \otimes D 2$. Let $\Sigma_{s}$ be a possible $s \times s$ principle submatrix of such a discrepancy matrix, call it $\Delta$. All entries in $\Delta$, and consequently in $\Sigma_{s}$, must be in $\{-1,0,1\}$ by virtue of corollary 6 . And as $\Delta$ is symmetric with zero diagonal, $\Sigma_{s}$ is necessarily so as well. $\Sigma_{s}$ is said to be feasible if

$$
\max _{w}\left[1-\frac{\left(\sum w_{i}\right)^{2}}{v}\right]^{-1} w^{T} \Sigma_{s} w \leq x_{\bar{d} 1}
$$

where $x_{\bar{d} 1}$ is the largest eigenvalue of $D 2$. This simply says that $\Sigma_{s}$ may be a principle submatrix for one of the discrepancy matrices sought. If the lefthand side of (10) is greater than $x_{\bar{d} 1}$, then $\Sigma_{s}$ is eliminated from consideration (is infeasible) by lemma 4.

Given feasible $\Sigma_{s}$, it is either itself a discrepancy matrix, or some of its rows have nonzero sums. In the latter case, fix any row ( $i$, say) that does not sum to zero. Create a vector $h_{s \times 1}$ with all entries from $\{-1,0,1\}$ and with $h_{i}=-1$ or 1 as the fixed row $i$ has positive or negative sum. Now create a new matrix $\Sigma_{s+1}$ by

$$
\Sigma_{s+1}=\left(\begin{array}{cc}
\Sigma_{s} & h \\
h^{\prime} & 0
\end{array}\right)
$$


If $\Sigma_{s+1}$ is a discrepancy matrix, its largest eigenvalue can be compared to $x_{\bar{d} 1}$. If not, it can be checked for feasibility. If feasible it can be extended to some $\Sigma_{s+2}$, the evaluation repeated, and so on. This produces the following constructive algorithm for discrepancy matrices:

1. For a convenient value of $s$, create an exhaustive list of nonequivalent (under row/column permutation) $s \times s$ matrices that could serve as leading diagonal submatrices for a discrepancy matrix. Each matrix must be symmetric with zero diagonal and have all off-diagonal elements in $\{-1,0,1\}$.

2. If any matrix in the list is a discrepancy matrix (has zero row/column sums), remove it from the list and calculate its largest eigenvalue. If larger than $x_{\bar{d} 1}$, the matrix is dropped from consideration. Otherwise, it is one of the matrices sought.

3. Evaluate each matrix in the list for feasibility using (10). Discard all infeasible matrices.

4. If the list is nonempty, pick a remaining submatrix and select a row with nonzero sum. Construct all $3^{s-1}$ extension vectors $h_{s \times 1}$ with the selected row position fixed at $-1 \times$ ( $\operatorname{sign}$ of row sum), and from these all $3^{s-1}$ extended submatrices of order $s+1$. Repeat for each list member.

5. Replace the list of order $s$ matrices with the list of all the order $s+1$ matrices created. Return to step 2 with $s$ replaced by $s+1$.

Step 2 says that the algorithm will not find discrepancy matrices containing principle submatrices which are also discrepancy matrices. This is the point of lemma 7: the larger $\Delta$ cannot improve on (in fact is Schur-inferior to) a discrepancy submatrix $\Sigma_{s}$. The algorithm will find every discrepancy matrix that does not contain a discrepancy matrix submatrix; these "parts" can be assembled into larger discrepancy matrices if desired. Note that in the partitioning of lemma $7,\left(\begin{array}{cc}0 & \Delta_{d 12} \\ \Delta_{d 21} & \Delta_{d 22}\end{array}\right)$ is also a discrepancy matrix, as is $\left(\begin{array}{cc}0 & \Delta_{d 12} \\ \Delta_{d 21} & 0\end{array}\right)$ should $\Delta_{d 22}$ be. The process of "fixing" a row, described in step 4, with no loss of generality can be done for any one row not currently summing to zero, since the fixed position value can always be achieved by row/column permutation of $\Delta$ with the first $s$ row/columns $\left(\Sigma_{s}\right)$ fixed.

To keep the list of submatrices from becoming explosively large, a few size-reducing measures are advisable. First, in step 4, symmetries among the rows can be taken advantage of to avoid 
creating the full set of $3^{s-1}$ extensions. For instance, if all permutations of rows/columns 1, 2, and 3 produce the same matrix, then only 10 choices for positions $(1,2,3)$ in $h$ need be considered, not 27. Second, in step 5, an equivalence screen can be performed on the new list before returning to step 2. It is not uncommon to have different submatrices extend to equivalent candidates. Finally, for each submatrix the choice in step 4 of which row to "fix" can significantly change the number of feasible extensions produced. Experience with the algorithm helps in making good choices.

As described the constructive algorithm is for a given $v$. But notice that the lefthand side of (10), when considered as a function of $v$, is decreasing. Consequently, if the algorithm is completed for value $v^{*}$, the results are correct for all $v \leq v^{*}$ (omitting, of course, any discrepancy matrices of order larger than the desired $v$ ). The downside is that larger $v^{*}$ can increase the number of submatrices surviving the feasibility check at each order, possibly becoming too large to handle.

The remainder of this section will describe the results found by completing the algorithm for 100 treatments. The problem was further divided into three disjoint cases (cases 1 and 2 place clear restrictions on choice of extension vectors $h$ in step 4$)$ :

Case 1: There is at most a single one in every row. With $s=3$ the only submatrix for starting the constructive algorithm is:

$$
\begin{array}{rrr}
0 & 1 & -1 \\
1 & 0 & 0 \\
-1 & 0 & 0
\end{array}
$$

This submatrix is not subjected to the initial step 2 screen, for there cannot exist a design for which it is a discrepancy matrix.

Case 2: There is at least one row with exactly two ones but no row with three or more ones. Taking $s=3$ and without loss of generality assuming first row and column as shown, there are three nonequivalent submatrices:

\begin{tabular}{rrrrrrrrr} 
& \multicolumn{1}{c}{$(i)$} & \multicolumn{3}{c}{$($ ii $)$} & \multicolumn{3}{c}{$($ iii $)$} \\
0 & 1 & 1 & 0 & 1 & 1 & 0 & 1 & 1 \\
1 & 0 & -1 & 1 & 0 & 0 & 1 & 0 & 1 \\
1 & -1 & 0 & 1 & 0 & 0 & 1 & 1 & 0
\end{tabular}


Case 3: There is at least one row with three or more ones. Taking the initial value $s=4$, this leads to 10 nonequivalent $4 \times 4$ submatrices which without loss of generality have first row and column as shown in the three matrices below. Of these, seven are immediately discarded as infeasible (do not survive step 3). The three remaining are:

$(i)$

$\begin{array}{rrrrrrrrrrrr}0 & 1 & 1 & 1 & 0 & 1 & 1 & 1 & 0 & 1 & 1 & 1 \\ 1 & 0 & -1 & -1 & 1 & 0 & -1 & -1 & 1 & 0 & -1 & 0 \\ 1 & -1 & 0 & -1 & 1 & -1 & 0 & 0 & 1 & -1 & 0 & 0 \\ 1 & -1 & -1 & 0 & 1 & -1 & 0 & 0 & 1 & 0 & 0 & 0\end{array}$

The constructive algorithm was run to completion (see below for a wrinkle in case 3 ) for each case, for $v=100$ and comparing to $x_{\bar{d} 1}=1.73205$ of discrepancy matrix D2 of Reck and Morgan (2005). Case 1 quickly resolves to a single discrepancy matrix, which in fact is $D 2$. For case 2, submatrix $(i i i)$ is infeasible and submatrix $(i i)$ has no feasible extensions after $6 \times 6$ (in our sequence of choices for fixed positions; other choices could conceivably lead to either fewer or more loops through the algorithm). Case $2(i)$ produces exactly two discrepancy matrices, one superior to $D 2$ and one its equal; see Table 1.

Table 1: Constructed Discrepancy Matrices

$\begin{array}{rrrrrrrrrrrrrrrr}0 & 1 & 1 & -1 & -1 & 0 & 0 & 0 & 1 & 1 & -1 & 0 & 0 & -1 & 0 & 0 \\ 1 & 0 & -1 & 1 & 0 & -1 & 0 & 1 & 0 & -1 & 0 & 0 & 0 & 0 & 0 & 0 \\ 1 & -1 & 0 & 0 & 1 & 0 & -1 & 1 & -1 & 0 & 0 & 0 & 0 & 0 & 0 & 0 \\ -1 & 1 & 0 & 0 & 0 & 1 & -1 & -1 & 0 & 0 & 0 & 1 & 1 & -1 & 0 & 0 \\ -1 & 0 & 1 & 0 & 0 & -1 & 1 & 0 & 0 & 0 & 1 & 0 & -1 & 0 & 0 & 0 \\ 0 & -1 & 0 & 1 & -1 & 0 & 1 & 0 & 0 & 0 & 1 & -1 & 0 & 0 & 0 & 0 \\ 0 & 0 & -1 & -1 & 1 & 1 & 0 & -1 & 0 & 0 & -1 & 0 & 0 & 0 & 1 & 1 \\ & & 0 & 0 & 0 & 0 & 0 & 0 & 0 & 1 & 0 & -1 \\ \text { max eigenvalue }=1.69202 & 0 & 0 & 0 & 0 & 0 & 0 & 1 & -1 & 0 \\ & \end{array}$


Case 3 was more problematic in the many competitors produced and in an additional argument needed to bring the constructive algorithm to completion. Let $e_{j}$ denote a $j \times 1$ vector with one in the $j^{\text {th }}$ position and otherwise 0 . As usual $I_{j}$ is the order $j$ identity matrix, and $J_{j_{1}, j_{2}}$ is a $j_{1} \times j_{2}$ matrix of ones. The argument requires two partitioned matrices, call them $D_{t}$ and $D_{t}^{0}$, with exact form depending on the parity of $t$ :

$$
\begin{aligned}
& D_{2 n}=\left(\begin{array}{c|c}
I_{n}-J_{n, n} & J_{n, n} \\
\hline J_{n, n} & I_{n}-J_{n, n}
\end{array}\right) \quad D_{2 n}^{0}=\left(\begin{array}{c|c|c}
\frac{I_{n-1}-J_{n-1, n-1}}{J_{n, n-1}} & J_{n-1, n} & -e_{n}-J_{n, n} \\
\hline-e_{2 n-1}^{\prime} & 0
\end{array}\right)
\end{aligned}
$$

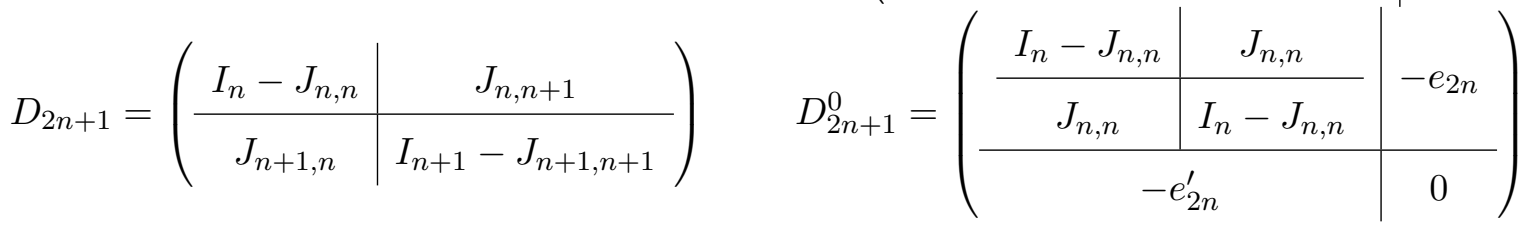

Though requiring lengthy runs, cases $3(i i)$ and 3(iii) both complete the constructive algorithm without producing a discrepancy matrix. With this information in hand, every feasible submatrix arising from case $3(i)$ at any step containing either $3(i i)$ or $3(i i i)$ as a submatrix can be eliminated immediately (as it has already been proven that their paths could not be completed to an acceptable discrepancy matrix). This is useful in culling a great number of possibilities, and by the $11 \times 11$ stage of our run only two matrices remained: $D_{11}$ and $D_{11}^{0}(n=5$ in the display above). Fixing the last row of $D_{11}^{0}$, it produces no feasible extensions. Fixing the first row of $D_{11}$ produces two feasible extensions: $D_{12}$ and $D_{12}^{0}$. Fixing the last row of $D_{12}^{0}$, it produces no feasible extensions, while fixing the first row of $D_{12}$ produces two feasible extensions: $D_{13}$ and $D_{13}^{0}$.

Lemma 8 For any $t \geq 11$ and $v \leq 100, D_{t}$ admits at most two feasible extensions, $D_{t+1}$ and $D_{t+1}^{0}$, when fixing its first row. Upon fixing its last row, $D_{t}^{0}$ admits no feasible extensions.

Proof By induction. Assume the statement is true up to given $t$ and suppose $D_{t+1}$ is feasible (otherwise this branch of the constructive algorithm has stopped). Consider extending $D_{t+1}$ to

$$
E_{t+2}=\left(\begin{array}{cc}
D_{t+1} & h \\
h^{\prime} & 0
\end{array}\right)
$$

where position 1 of the $(t+1) \times 1$ extension vector $h$ is fixed at -1 . If the remaining positions in $h$ are all 0 , then $E_{t+2}$ is equivalent to $D_{t+2}^{0}$. If $h$ is -1 in all of its first int $\left(\frac{t+1}{2}\right)$ positions, and 
1 elsewhere, then it is equivalent to $D_{t+2}$. With this in mind, delete two rows and columns from $E_{t+2}$, one chosen from positions $2,3, \ldots, \operatorname{int}\left(\frac{t+1}{2}\right)$, and one from the remaining positions save the last. This produces

$$
E_{t}^{*}=\left(\begin{array}{cc}
D_{t-1} & h^{*} \\
h^{* \prime} & 0
\end{array}\right)
$$

Now $E_{t}^{*}$ is a submatrix of the feasible matrix $D_{t+1}$, so is itself feasible. It is also an extension of $D_{t-1}$ with first row fixed at -1 , so by the induction hypothesis there are only two possibilities for the vector $h^{*}$. Since the same possibilities occur regardless of which pair of rows (one from each set of positions) are deleted, $h$ has only the two possible values already mentioned, giving the result. A similar argument quickly gives the conclusion when extending $D_{t+1}^{0}$.

The consequence of lemma 8 is that case 3 can produce no discrepancy matrices (neither $D_{t}$ nor $D_{t}^{0}$ is a discrepancy matrix for any $t$ ).

TheOREM 9 The first discrepancy matrix in Table 1 is E-best for all irregular BIBD settings with with up to 100 treatments.

\section{E-Optimality in D $(15,21,5)$}

The smallest irregular BIBD setting in terms of $k$ is $\mathrm{D}(15,21,5)$. Reck and Morgan (2005) executed a search to determine A- and D-optimal designs in this setting. Adopting their techniques to focus on the first discrepancy matrix in Table 1 produces the E-optimal design $d^{*}$ in Table 2.

Table 2: An E-optimal Design In $D(15,21,5)$

$\begin{array}{rrrrrrrrrrrrrrrrrrrrr}1 & 1 & 2 & 4 & 5 & 2 & 1 & 5 & 1 & 4 & 3 & 2 & 1 & 3 & 4 & 1 & 3 & 3 & 2 & 2 & 1 \\ 2 & 6 & 3 & 5 & 6 & 4 & 3 & 9 & 2 & 7 & 5 & 6 & 4 & 7 & 8 & 10 & 6 & 4 & 7 & 5 & 5 \\ 3 & 7 & 8 & 6 & 8 & 9 & 7 & 11 & 6 & 9 & 8 & 11 & 8 & 10 & 12 & 11 & 9 & 6 & 8 & 7 & 9 \\ 4 & 8 & 9 & 7 & 10 & 10 & 11 & 12 & 10 & 10 & 10 & 12 & 11 & 12 & 13 & 13 & 13 & 13 & 13 & 13 & 14 \\ 5 & 9 & 11 & 11 & 12 & 12 & 12 & 13 & 14 & 15 & 15 & 15 & 14 & 14 & 14 & 15 & 14 & 15 & 15 & 14 & 15\end{array}$

Is this the unique E-optimal design? Probably not. There may be nonisomorphic designs with the same discrepancy matrix. And there may well be E-equal designs in $\mathrm{D}(15,21,5)$ with discrepancy matrix $I_{2} \otimes \Delta_{d^{*}}$. 


\section{Summary}

This research began as an attempt to determine an E-optimal design in $D(15,21,5)$. It quickly became apparent that, even for $v$ as small as 15, enumeration of all discrepancy matrices was impossible, leading to development of the feasibility bound (10) and the constructive algorithm. The realization that the lefthand side of (10) is decreasing in $v$ then meant that results were not constrained by a "one design setting at a time" approach. That fact has allowed us to produce results for all $v$ up to 100 .

The results tell those seeking E-optimal designs in irregular BIBD settings what structures to look for, at least for $v \leq 100$. The first discrepancy matrix in Table 1 is preferred. Should no design with this concurrence structure be found, kronecker products of that matrix with the identity can be tried; the desirability of this may be limited if other criteria such as A are also of some interest, as A-efficiency is generally expected to decline with increasing discrepancy value. The next best choices are $D 2$ (for which no design exists in $\mathrm{D}(15,21,5)$ ) and the second matrix in Table 1.

Unless a great many concurrence structures fail to exist, it appears unlikely that A- and Eoptimal designs will coincide in irregular BIBD settings. We find this very surprising. It is, in some sense, an artifact of difficulties imposed by the nonexistence of symmetry.

\section{ACKNOWLEDGEMENT}

J. P. Morgan was supported by National Science Foundation grant DMS01-04195. Brain Reck was supported by National Institute of Mental Health grant T32 MH20053. 


\section{A E-ORDERING OF DISCREPANCY MATRICES}

The 51 discrepancy matrices with discrepancy values $\delta_{d} \leq 5$ are ordered here by E-value. The actual matrices may be found in Reck and Morgan (2005). Those authors define the concurrence range $l_{d}$, while $w_{d}$ is the treatment deficiency of Hedayat, Stufken, and Zhang (1995).

$\begin{array}{clllll}\text { rank } & \text { Matrix } & \delta_{d} & l_{d} & w_{d} & x_{d 1} \\ 1 & \text { D2 } & 3 & 2 & 4 & 1.73205 \\ 2 & \text { D13 } & 5 & 2 & 4 & 1.87939 \\ 3 & \text { D23 } & 5 & 2 & 5 & 1.90211 \\ 4 & \text { D5 } & 4 & 2 & 4 & 1.93543 \\ 5 & \text { D1 } & 2 & 2 & 2 & 2.00000 \\ 5 & \text { D4 } & 4 & 2 & 3 & 2.00000 \\ . & \text { D6 } & 4 & 2 & 4 & 2.00000 \\ . & \text { D7 } & 4 & 2 & 4 & 2.00000 \\ . & \text { D14 } & 5 & 2 & 3 & 2.00000 \\ . & \text { D15 } & 5 & 2 & 3 & 2.00000 \\ 5 & \text { D24 } & 5 & 2 & 5 & 2.00000 \\ 12 & \text { D20 } & 5 & 2 & 5 & 2.13452 \\ 13 & \text { D3 } & 3 & 2 & 3 & 2.23607 \\ . & \text { D16 } & 5 & 2 & 4 & 2.23607 \\ . & \text { D26 } & 5 & 2 & 5 & 2.23607 \\ 13 & \text { D29 } & 5 & 2 & 5 & 2.23607 \\ 17 & \text { D17 } & 5 & 2 & 4 & 2.29240 \\ 18 & \text { D25 } & 5 & 2 & 5 & 2.30278 \\ 19 & \text { D27 } & 5 & 2 & 5 & 2.35829 \\ 20 & \text { D21 } & 5 & 2 & 3 & 2.37720 \\ 21 & \text { D28 } & 5 & 2 & 3 & 2.37951 \\ 22 & \text { D12 } & 4 & 3 & 3 & 2.41421 \\ 23 & \text { D41 } & 5 & 2 & 5 & 2.42534 \\ 24 & \text { D8 } & 4 & 2 & 3 & 2.44949 \\ 25 & \text { D22 } & 5 & 2 & 4 & 2.45585 \\ 26 & \text { D10 } & 4 & 2 & 4 & 2.47283 \\ 27 & \text { D30 } & 5 & 2 & 3 & 2.52434 \\ 28 & \text { D19 } & 5 & 2 & 3 & 2.52543 \\ 29 & \text { D18 } & 4 & 3 & 4 & 2.56155 \\ . & \text { D31 } & 5 & 2 & 3 & 2.56155 \\ . & \text { D32 } & 5 & 2 & 3 & 2.56155 \\ . & \text { D33 } & 5 & 2 & 3 & 2.56155 \\ . & \text { D44 } & 5 & 2 & 3 & 2.56155 \\ 29 & \text { D45 } & 5 & 2 & 4 & 2.56155 \\ 35 & \text { D34 } & 5 & 2 & 3 & 2.61050 \\ 36 & \text { D35 } & 5 & 2 & 3 & 2.64575 \\ 37 & \text { D42 } & 5 & 2 & 3 & 2.69963 \\ 38 & \text { D36 } & 5 & 2 & 4 & 2.71519 \\ 39 & \text { D37 } & 5 & 2 & 3 & 2.79793 \\ 39 & \text { D38 } & 5 & 2 & 3 & 2.79793 \\ 41 & \text { D46 } & 5 & 2 & 3 & 2.81361 \\ 42 & \text { D9 } & 4 & 2 & 2 & 2.82843 \\ 43 & \text { D43 } & 5 & 2 & 4 & 2.85323 \\ 44 & \text { D47 } & 5 & 2 & 4 & 2.89511 \\ 45 & \text { D11 } & 4 & 2 & 3 & 2.90321 \\ 46 & \text { D39 } & 4 & 3 & 3 & 3.00000 \\ . & \text { D40 } & 4 & 3 & 3 & 3.00000 \\ 46 & \text { D48 } & 4 & 3 & 4 & 3.00000 \\ 49 & \text { D50 } & 5 & 2 & 4 & 3.04892 \\ 50 & \text { D49 } & 5 & 2 & 3 & 3.15633 \\ 51 & \text { D51 } & 4 & 3 & 3 & 3.44949 \\ & & & & & \end{array}$




\section{REFERENCES}

Hanani, H. (1961). The existence and construction of balanced incomplete block designs. Ann. Statist. 32, 361-386.

Hedayat, A. S., Stufken J., and Zhang, W. G. (1995). Contingently and virtually balanced incomplete block designs and their efficiencies under various optimality criteria. Statistica Sinica $\mathbf{5}$, $575-591$.

JACroux, M. (1980a). On the determination and construction of E-optimal block designs with unequal number of replicates. Biometrika 67, 661-667.

Jacroux, M. (1980b). On the E-optimality of regular graph designs. J. R. Statist. Soc. B. 42, 205-209.

Kiefer, J. (1975). Construction and optimality of generalized Youdan designs. In A Survey of Statistical Designs and Linear Models, Ed. J. N. Srivastava, pp. 333-353. Amsterdam, North Holland. Marshall, A. W. and Olkin, I. (1979). Inequalities: Theory of Majorization and Its Applications. Academic Press, Inc., New York.

Morgan, J. P. and Srivastav, S. K. (2000). On the type-I optimality of nearly balanced incomplete block designs with small concurrence range. Statistica Sinica 10, 1091-1116.

Nandi, H. K. (1945). On the relation between certain types of tactical configurations. Bull. Calcutta Math. Soc. 37, 92-94.

Reck, B. H. And Morgan, J. P. (2005). Optimal design in irregular BIBD settings. J. Statist. Plann. Inf. 129, 59-84. 